\title{
Methyl-Coenzyme M Reductase and Its Post-translational Modifications
}

\author{
Hao Chen ${ }^{1}$, Qinglei Gan ${ }^{2}$ and Chenguang Fan ${ }^{1,2 *}$

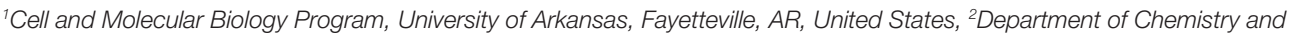 \\ Biochemistry, University of Arkansas, Fayetteville, AR, United States
}

The methyl-coenzyme M reductase (MCR) is a central enzyme in anaerobic microbial methane metabolism, which consists of methanogenesis and anaerobic oxidation of methane (AOM). MCR catalyzes the final step of methanogenesis and the first step of $\mathrm{AOM}$ to achieve the production and oxidation of methane, respectively. Besides a unique nickel tetrahydrocorphinoid (coenzyme F430), MCR also features several unusual posttranslational modifications (PTMs), which are assumed to play important roles in regulating MCR functions. However, only few studies have been implemented on MCR PTMs. Therefore, to recapitulate current knowledge and prospect future studies, this review summarizes and discusses studies on MCR and its PTMs.

OPEN ACCESS

Edited by:

Yuchen Liu,

ExxonMobil, United States

Reviewed by: Zhe Lyu,

Baylor College of Medicine,

United States

Biswarup Mukhopadhyay,

Virginia Tech, United States

${ }^{*}$ Correspondence: Chenguang Fan cf021@uark.edu

Specialty section: This article was submitted to Microbiological Chemistry and Geomicrobiology,

a section of the journal

Frontiers in Microbiology

Received: 30 June 2020 Accepted: 11 September 2020 Published: 09 October 2020

Citation:

Chen H, Gan Q and Fan C (2020) Methyl-Coenzyme M Reductase and Its Post-translational Modifications.

Front. Microbiol. 11:578356.

doi: 10.3389/fmicb.2020.578356
Keywords: methyl-coenzyme M reductase, methanogenesis, post-translational modification, methylation, thioamidation, anaerobic oxidation of methane, anaerobic methanotrophic archaea, methanogenic archaea

\section{INTRODUCTION}

Anaerobic microbial methane metabolism is one key part of the global carbon cycle, which controls the methane emission to the atmosphere (Kirschke et al., 2013; Evans et al., 2019). Methanogenic archaea and anaerobic methanotrophic archaea (ANME) in anoxic environments are the major groups involved in microbial methane metabolism (Enzmann et al., 2018). During methanogenesis, methanogenic archaea use the end products of fermentation to produce methane anaerobically (Sieber et al., 2012; Vanwonterghem et al., 2016; Lyu et al., 2018b; Evans et al., 2019). There are three major pathways of methanogenesis, namely hydrogenotrophic, aceticlastic, and methylotrophic (Lyu and Whitman, 2019). On the other hand, ANME treat $\mathrm{CH}_{4}$ as a carbon source and get energy from oxidizing it into $\mathrm{CO}_{2}$, which is so-called anaerobic oxidation of methane (AOM; Orphan et al., 2001; Scheller et al., 2016; Timmers et al., 2017). Methanogenesis and AOM are thought to be reverse to each other based on the fact that the methyl-coenzyme M reductase (MCR) is involved in both methanogenesis and AOM, and its gene sequences are similar in both methanogenic archaea and ANME (Knittel and Boetius, 2009; Prakash et al., 2014; Ragsdale, 2014; Wang et al., 2014; Wongnate and Ragsdale, 2015). Previous studies have explored this enzyme from phylogeny to structures, reactions, and functions (Mansoorabadi et al., 2017; Thauer, 2019). As one remarkable feature of MCRs, coenzyme F430 located in the active site has been proved to be crucial, and focuses have been put on its reaction mechanism and activation (Wongnate and Ragsdale, 2015; Wongnate et al., 2016; Lyu et al., 2018a). Besides coenzyme F430, unusual post-translational modification (PTM) is identified as another remarkable feature of MCR (Kahnt et al., 2007; Mansoorabadi et al., 2017; Nayak et al., 2020). However, the biosynthesis and functions of MCR PTMs remain largely unclear. Here, we provide an introduction of $\mathrm{MCR}$, and then on recent studies on PTMs of MCR. 


\section{METHYL-COENZYME M REDUCTASE}

MCR is the central enzyme of both methanogenesis and AOM. MCRI and MCRII are the common isozymes of MCR found in methanogenic archaea. They were firstly distinguished in Methanothermobacter marburgensis by anion exchange chromatography (Rospert et al., 1990). Besides M. marburgensis, many other species of Methanobacteriales and some species of other genera such as Methanococcales have been found to contain both MCRI and MCRII (Wagner et al., 2017). Furthermore, based on phylogenetic reconstruction of MCRs, Wagner et al. demonstrated a new type of MCR (highly structurally similar to MCRI and MCRII) exclusively from Methanococcales, so called MCRIII (Wagner et al., 2017). But, there is only one type of MCR found in ANME (Thauer, 2019).

\section{Structure Features of MCRs}

MCRs contain three different subunits, $\alpha$ (McrA), $\beta$ (McrB), and $\gamma$ (McrG). And the high-resolution X-ray structures showed that MCRs usually function as a dimer of heterotrimers $(\alpha 2 \beta 2 \gamma 2)$ with the nickel tetrahydrocorphinoid (coenzyme F430) at its active sites (Ermler et al., 1997; Wagner et al., 2017). In each heterotrimer $(\alpha \beta \gamma)$, three subunits are tightly associated with each other, leaving only a 50 Å long hydrophobic channel, which starts from the surface and ends with a narrow pocket containing a coenzyme F430 in the active site (Ermler et al., 1997; Thauer, 2019). Similarly, an X-ray structure of the MCR from an ANME-1 archaea also showed a dimer of a heterotrimer structure, and further analysis of this structure suggested that methanogenic and methanotrophic MCRs share the same substrates (Shima et al., 2012). However, there are also paradoxes to link methanogenic MCRs to methanotrophic MCRs, such as a coenzyme F430 modified with a methylthio group and different PTM patterns (Shima et al., 2012; Mansoorabadi et al., 2017).

\section{Assembly of MCRs}

The MCR complex is encoded by mcrBDCGA operon ( $m c r C$ is absent in operon for MCRII), and the gene order is conserved in both methanogenic archaea and ANME (Thauer, 2019). The assembly and maturation of the MCR complex has been recently studied, and a co-translated "order assembly" model was hypothesized to explain the assembly process of MCR complex. In this model, McrB is first expressed, and forms an initial complex with McrD. The complex McrBD then associates McrG and McrA when they are translated. The McrD unit is possibly lost or weakly associated after facilitating PTMs in active site and the binding of coenzyme F430 to the modified complex (Lyu et al., 2018a). Although this model provided a possible pathway of MCR complex assembly, it was speculative, and more future studies are needed to explore the specific function of the McrD unit and its interactions with the functional part McrABG.

\section{Activation of MCR}

The activity of MCR depends on its unique nickel-containing coenzyme F430. The nickel in coenzyme F430 can be in three different oxidation states: redox-active $\mathrm{Ni}(\mathrm{I})$, inactive $\mathrm{Ni}(\mathrm{II})$, and $\mathrm{Ni}(\mathrm{III})$ (Wongnate and Ragsdale, 2015). It was wellestablished that to initiate catalysis, the MCR complex must be in its active state, EPR-active $\left\{\mathrm{MCR}_{\text {red1 }}[\mathrm{Ni}(\mathrm{I})-\mathrm{F} 430]\right\}$, where the nickel is in the 1+ oxidation state (Becker and Ragsdale, 1998; Thauer, 2019). M. marburgensis has a strong $\mathrm{MCR}_{\mathrm{red} 1}$ [Ni(I)-F430] signal when incubated with $100 \% \mathrm{H}_{2}$ or $\mathrm{CO}$ (Rospert et al., 1991; Zhou et al., 2013). In addition, in the presence of reductant $\mathrm{Ti}(\mathrm{III})$ citrate, the $\mathrm{MCR}_{\mathrm{ox} 1}$ [high spin $\mathrm{Ni}(\mathrm{II})$ thiyl-radical] form (inactive) could be converted to $\mathrm{MCR}_{\text {red1 }}$ [Ni(I)-F430] form (active; Goubeaud et al., 1997). Later, Prakash et al. performed an in vitro cell free activation system of MCR in the presence of dithiothreitol and protein components A2, an ATP carrier, and A3a (Prakash et al., 2014). It indicated the possible functions of protein components A2 and A3a. A2 with two ATP binding domains could serve as a carrier for ATP to Fe protein homolg and help ATP hydrolysis. A3a was a multienzyme complex containing $\mathrm{mcrC}$ gene product, and protein components involved in heterodisulfide (HDS) reductase coupled electron bifurcation (Prakash et al., 2014). However, the detailed function of each component in $\mathrm{A} 3 \mathrm{a}$ in $\mathrm{MCR}$ activation still remains unclear. Furthermore, this study also demonstrated that the presence of CoM-S-S-CoB (HDS) could promote the inactivation of the enzyme (Prakash et al., 2014).

\section{Reaction Mechanisms of MCRs}

The reaction catalyzed by MCRs involves two substrates (the methyl donor methyl-CoM and the electron donor $\mathrm{CoB}$ ) and two products $\left(\mathrm{CH}_{4}\right.$ and CoM-S-S-CoB $)$. In the methanogenesis process, the final reaction occurs when substrates bind to the substrate channel at the active site, where methyl-CoM is proximal to the nickel in coenzyme F430 (Wongnate et al., 2016). However, the exact mechanism to explain the reaction at the active site has not been fully solved. There are three main proposed mechanisms: mechanism I involves a generation of a methyl-Ni(III) intermediate (Horng et al., 2001); mechanism II assumes the production of a $\mathrm{Ni}(\mathrm{II})$-thiolate and a methyl radical (Scheller et al., 2013; Wongnate et al., 2016); and mechanism III indicates the existence of intermediates, a highly reactive methyl anion and $\mathrm{Ni}(\mathrm{III})-\mathrm{SCoM}$ (Harmer et al., 2005). A recent study conducted by Wongnate and Ragsdale elucidated the order of reactions at the active site of MCR based on the kinetic approach (Wongnate and Ragsdale, 2015). The binding of methyl-CoM to nickel in coenzyme F430 triggers the movement of $\mathrm{CoB}$, then generates the enzyme-substrate complex [CoB-MCR(NiI) $\cdot \mathrm{CH}_{3}-\mathrm{CoM}$ ], and releases $\mathrm{CH}_{4}$. Later, Wongnate and Ragsdale applied transient kinetic, spectroscopic, and computational approaches and obtained results more consistent with mechanism II, the methyl radical mechanism (Wongnate et al., 2016). On the other direction, although studies also suggested that the first step of AOM could also be the ratelimiting step (Scheller et al., 2010), the reaction mechanism of MCR in ANME was rarely studied, either from the kinetic or structural aspect. 


\section{POST-TRANSLATIONAL MODIFICATIONS OF MCRs}

Unusual PTMs are another remarkable feature of MCRs. The first observation of PTMs in MCR dated back to 1997 when Ermler et al. determined the first high-resolution crystal structure of MCRI in a Methanobacterium thermoautotrophicum. Their study demonstrated the existence of five PTMs at the active sites of MCR (Ermler et al., 1997). So far, 1- $N$-methylhistidine, $S$-methylcysteine, 2-(S)-methylglutamine, 5-(S)-methylarginine, thioglycine, didehydroaspartate, and 6-hydroxy-tryptophan have been found in methanogenic MCRs (Ermler et al., 1997; Selmer et al., 2000; Wagner et al., 2016, 2017; Mansoorabadi et al., 2017). Among these PTMs, arginine methylation can be found in all methanogenic archaea under laboratory conditions but not ANME like ANME-1 (Kahnt et al., 2007). Cysteine methylation is in a low abundance or absent in many methanogenic archaea, such as M. maripaludis. Glutamine methylation is absent in Methanosarcina barkeri (Selmer et al., 2000; Lyu et al., 2018a). Didehyroaspartate is absent in Methanothermobacter wolferi (Wagner et al., 2016). Although methanotrophic MCRs share two conserved PTMs, 1-N-methylhistidine and thioglycine with methanogenic archaea, they harbor their own PTMs, 7-hydroxy-tryptophan and S-oxymethionine (Shima et al., 2012; Wagner et al., 2017; Figure 1; Table 1).

\section{Methylation}

Widely occurring at the active site of methanogenic MCRs, methylation has been identified at four different amino acid side chains, including histidine, arginine, glutamine, and cysteine. These four types of methylation are also the first group of PTMs found in MCRs (Ermler et al., 1997). Several studies have been implemented to study their biosynthesis. Firstly, Selmer et al. ruled out the possibility that the methyl groups are donated from methyl-CoM during the reaction at the active site of MCRs, and they confirmed that $S$-adenosylmethionine (SAM) was the most likely methyl donors. This was based on the observation that after culturing $M$. thermoautotrophicum in the media with L-(methyl- $\left.\mathrm{D}_{3}\right)$ methionine, the $\mathrm{D}_{3}$-labeled pattern was the same as two methyl groups known to be introduced by SAM in coenzyme F430 while the SAM was not used as the carbon source of methanogenesis and converted into methane (Selmer et al., 2000). This study also indicated that the biosynthesis of $1-N$-methylhistidine and $S$-methylcysteine was chemically possible to process via the methyl group transfer from SAM to substitute the hydrogen of a thiol group or a ring nitrogen (nucleophilic substrates). However, this mechanism could not explain the occurrence of 2-(S)-methylglutamine and 5-(S)-methylarginine due to the low nucleophilicity of the target carbon (Selmer et al., 2000). Moreover, it was indicated that the biosynthesis of 1-N-methylhistidine and $S$-methylcysteine was probably catalyzed by SAM-dependent $N$ - and $S$-methyltransferases by which the methyl group is transferred to $N$ - or $S$-nucleophiles of histidine or cysteine via a $\mathrm{S}_{\mathrm{N}} 2$ reaction. The gene $(\mathrm{mcmA})$ encoding the SAM-dependent methyltransferase for cysteine methylation has been recently identified, and it has been indicated that cysteine methylation might play a role in adaption to mesophilic conditions (Nayak et al., 2020). However, 2-(S)-methylglutamine and 5-(S)-methylarginine could not be formed by this mechanism (Liscombe et al., 2012; Nayak et al., 2020).

To identify the mechanism for glutamine and arginine methylation, radical SAM methyltransferases were assumed, since they have the ability to transfer methyl groups to the electrophilic sp2- or sp3-carbon centers of substrates (Bauerle et al., 2015). Based on this hypothesis, methanogenesis marker protein 10 (Mmp10), a candidate of methyltransferase for 5-(S)-methylarginine was selected as the target to test. Methanosarcina acetivorans with the deletion of ma4551 (the gene for Mmp10) showed a loss of 5-(S)-methyl-arginine ${ }^{285}$ at the active site of MCR, and further investigation indicated that the lack of methylation in arginine ${ }^{285}$ could influence the thermal stability of MCR and also cause the growth defects under stress conditions, such as oxidative stress and elevated temperature stress (Deobald et al., 2018). Additionally, an in vitro assay of Mmp10 from $M$. acetivorans suggested that besides SAM, cobalamin was also a cofactor required for Mmp10 activity (Radle et al., 2019). Very recently, by knocking out $m m p X$ gene (encoding Mmp10 in M. maripaludis), Lyu et al. demonstrated a direct link between Mmp10 and arginine methylation based on the fact that the methylation of $\mathrm{Arg}^{275}$ was absent at the active site of MCR in mutant M. maripaludis. This study was the first to link MCR activity and a specific PTM. It also firstly demonstrates that a PTM could affect both methanogenesis and cell growth without the addition of stressor (Lyu et al., 2020). Furthermore, this study also indicated a high specificity of Mmp10 to McrA and the binding sites of cofactors, SAM, and cobalamin, in Mmp10 (Lyu et al., 2020). For glutamine methylation, although the putative cobalamin-dependent radical SAM enzyme has been proposed (Deobald et al., 2018), there has been no solid evidence. The functions of methylation in MCRs are not fully addressed with only few studies. Histidine methylation is theoretically thought to serve to position the imidazole ring that coordinates $\mathrm{CoB}$ (Grabarse et al., 2000), while arginine methylation at the active site of MCR could play multiple roles in the catalysis, assembly, or stability of the enzyme (Deobald et al., 2018; Lyu et al., 2020).

\section{Thioamidation}

Thioamides are very rare in nature, among which thioamidation of glycine in MCRs is the only example identified in proteins (Mahanta et al., 2018). Thioamidation commonly occurs in both methanogenic and methanotrophic MCRs. The biosynthesis of thioglycine has been hypothesized to involve two proteins, $\mathrm{YcaO}$ and TfuA. One recent study combined genetic approaches and mass spectrometry analyses to test the possible roles of $\mathrm{YcaO}$ and TfuA proteins in glycine thioamidation, proving that the removal of these two genes could cause the absence of thioamidation of glycin ${ }^{465}$ at the active site of MCR (Nayak et al., 2017). Later, Mahanta et al. used in vitro reconstitutions of thioamidation, using TfuA from M. acetivorans and $\mathrm{YcaO}$ 
A
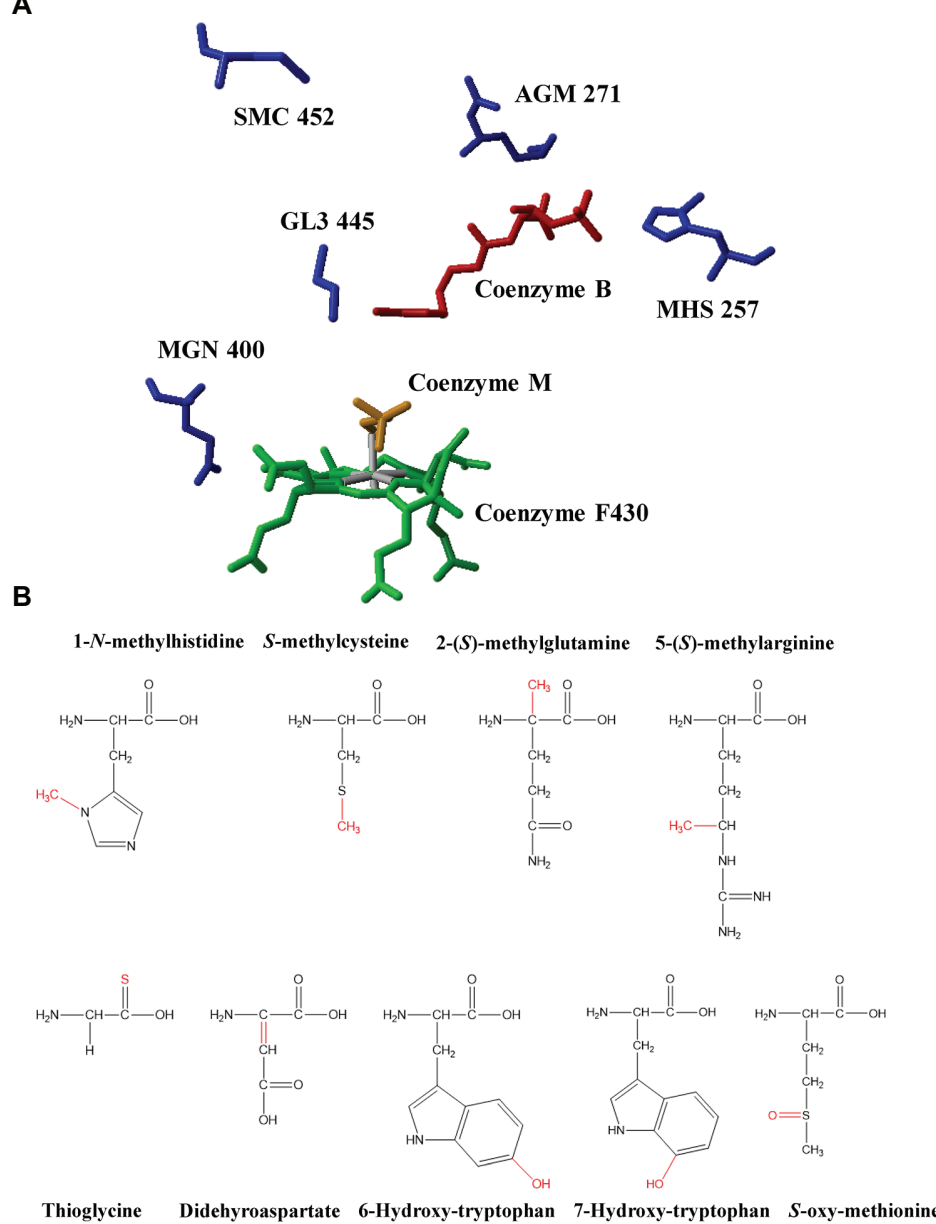

FIGURE 1 | (A) The active site of methyl-coenzyme M reductase (MCR) from Methanothermobacter marburgensis and identified post-translational modifications (PTMs; PDB ID: 1MRO, Ermler et al., 1997). Red: coenzyme B; Yellow: coenzyme M; Green: F430; AGM: 5-(S)-methyl-Arg ${ }^{271}$; MHS: 1-N-methyl-His ${ }^{257}$; GL3: thioGly ${ }^{445}$; MGN: 2-(S)-methyl-Gln ${ }^{400}$; and SMC: S-methyl-Cys ${ }^{452}$. (B) The chemical structures of PTMs identified in methyl-coenzyme M reductases from methanogenic archaea and anaerobic methanotrophic (ANME) archaea. Modifications were marked with red color.

from M. acetivorans, Methanopyrus kandleri, or Methanocaldococcus jannaschii, to demonstrate that thioamidation of glycine could proceed via backbone $O$-phosphorylation. This reaction is ATP-dependent and requires an external sulfide source (Mahanta et al., 2018). As for the function of thioamidation in MCRs, there are different opinions: (1) it facilitates the catalysis as an intermediate electron carrier during the reaction (Horng et al., 2001); (2) it reduces the pKa of sulfhydryl group to assist the deprotonation of $\mathrm{CoB}$ (Grabarse et al., 2001); and (3) it enhances the enzyme stability rather than facilitating catalysis (Nayak et al., 2017). Recently, to test the interactions between arginine methylation, cysteine methylation and thioamidation, Nayak et al. established knock-out strains with all possible combination, and observed that the $\Delta y c a O$ $t f u A / \triangle m c m A$ double mutant strain had most severe growth, while the one with triple deletion showed a faster growth, suggesting that unmodified $\operatorname{Arg}^{285}$ could alleviate changes brought by the absence of thioglycine and methyl-cysteine.
In addition, by phenotypic analyses, they showed that interactions between PTMs could cause unpredictable changes of the thermal stability of MCR, which might influence the activity and efficiency of MCRs (Nayak et al., 2020).

\section{Other PTMs}

Other PTMs in MCRs are rarely studied. Didehydroaspartate, the most recent PTM found in methanogenic MCRI and MCRII was identified to be adjacent to thioglycine based on mass spectrometry analyses and high-resolution X-ray crystallography (Wagner et al., 2016). This PTM was thought to be dispensable due to the fact that it was present in MCRs of M. marburgensis and M. barkeri, but not in MCR of Methanothermobacter wolfeii, which is phylogenetically closer to M. marburgensis. However, it was also shown that didehydroaspartate could slightly increase the catalytic efficiency of the enzyme (Wagner et al., 2016). Unlike didehydroaspartate, hydroxytryptophan was found in both methanogenic archaea and ANME. 7-hydroxy-tryptophan 
TABLE 1 | Recent findings on the biosynthesis and functions of PTMs in the active site of MCRs.

\begin{tabular}{|c|c|c|}
\hline PTMs & Biosynthesis & Functions \\
\hline \multicolumn{3}{|l|}{ Methanogens } \\
\hline 1-N-methylhistidine & SAM-dependent N-methyltransferase (No exact enzymes found yet) & $\begin{array}{l}\text { Might serve to position the imidazole that coordinates CoB } \\
\text { (Grabarse et al., 2000) }\end{array}$ \\
\hline S-methylcysteine & SAM-dependent S-methyltransferase (mcmA gene; Nayak et al., 2020) & $\begin{array}{l}\text { Might play a role in adaption to mesophilic conditions } \\
\text { (Nayak et al., 2020) }\end{array}$ \\
\hline 2-(S)-methylglutamine & Putative cobalamin-dependent radical SAM enzyme (No evidence yet) & Further studies needed \\
\hline 5-(S)-methylarginine & $\begin{array}{l}\text { Mmp10 protein (mmpX gene; Deobald et al., 2018; Radle et al., 2019; } \\
\text { Lyu et al., 2020) }\end{array}$ & $\begin{array}{l}\text { Play multiple roles in the catalysis, assembly or stability of MCR } \\
\text { (Lyu et al., 2020) }\end{array}$ \\
\hline Thioglycine & $\begin{array}{l}\text { YcaO and TfuA (ycaO and tfuA gene; Nayak et al., 2017; } \\
\text { Mahanta et al., 2018) }\end{array}$ & $\begin{array}{l}\text { Different opinions exist regarding to catalysis or stability of MCR } \\
\text { (Grabarse et al., 2001; Horng et al., 2001; Nayak et al., 2017) }\end{array}$ \\
\hline Didehydroaspartate & Further studies needed & $\begin{array}{l}\text { Found in MCRI and II, and slightly increased the catalytic efficiency } \\
\text { of the enzyme (Wagner et al., 2016) }\end{array}$ \\
\hline 6-hydroxy-tryptophan & Further studies needed & $\begin{array}{l}\text { Found in MCRIII, and might have equivalent role to the } \\
\text { didehydroaspartate in MCRI and II (Wagner et al., 2017) }\end{array}$ \\
\hline \multicolumn{3}{|l|}{ ANME } \\
\hline 1-N-methylhistidine & SAM-dependent $N$-methyltransferase (No exact enzyme found yet) & $\begin{array}{l}\text { Might serve to position the imidazole that coordinate CoB } \\
\text { (Grabarse et al., 2000) }\end{array}$ \\
\hline Thioglycine & $\begin{array}{l}\text { YcaO and TfuA (ycaO and tfuA gene; Nayak et al., 2017; } \\
\text { Mahanta et al., 2018) }\end{array}$ & $\begin{array}{l}\text { Different opinions exist regarding to catalysis or stability of MCR } \\
\text { (Grabarse et al., 2001; Horng et al., 2001; Nayak et al., 2017) }\end{array}$ \\
\hline 7-hydroxy-tryptophan & Further studies needed & $\begin{array}{l}\text { Assumed to compensate the absence of arginine methylation in } \\
\text { ANMEs (Shima et al., 2012) }\end{array}$ \\
\hline S-oxymethionine & Further studies needed & Further studies needed \\
\hline
\end{tabular}

at the active site of MCR from ANME-1 was assumed to compensate the absence of arginine methylation (Shima et al., 2012), while 6-hydroxy-tryptophan at the active site of MCRIII from Methanotorris formicicus was thought to have a role equivalent to the didehydroaspartate in MCRI and MCRII (Wagner et al., 2017). Till now, there are no proposed mechanisms for the biosynthesis of these PTMs yet, and no enzyme has been characterized to be related to them.

\section{DISCUSSION}

Recent studies have changed our view of the MCR from its evolutionary perspective to its two remarkable features, the conenzyme F430 and unusual PTMs at the active site. Recently, a study has provided supportive evidence for the methyl radical mechanism (Wongnate et al., 2016), which was only based on methanogenesis, so more future studies are necessary to explore the reaction mechanism for coenzyme 430 during the AOM. As for unusual PTMs, studies have focused on its biosynthesis and function. Histidine and cysteine are possibly catalyzed by SAM-dependent $\mathrm{N}$ - and S-methyltransferases, respectively, while arginine and glutamine seem to have different enzymatic methylation (Selmer et al., 2000). The methylation of cysteine and arginine have been shown to be related to a

\section{REFERENCES}

Bauerle, M. R., Schwalm, E. L., and Booker, S. J. (2015). Mechanistic diversity of radical S-adenosylmethionine (SAM)-dependent methylation. J. Biol. Chem. 290, 3995-4002. doi: 10.1074/jbc.R114.607044

Becker, D. F., and Ragsdale, S. W. (1998). Activation of methyl-SCoM reductase to high specific activity after treatment of whole cells with sodium sulfide. Biochemistry 37, 2639-2647. doi: 10.1021/bi972145x dedicated SAM-dependent (encoding by $m c m A$ ) and a potential radical SAM-dependent methyltransferase (Mmp10, encoding by $m m p X)$; however, the enzyme for histidine and glutamine modification still remains unknown (Deobald et al., 2018; Nayak et al., 2020). YcaO and TfuA were found to be involved in the biosynthesis of thioglycine. An elucidation of the mechanisms or enzymes for other PTMs like didehydroaspartate and hydroxytryptophan at the active site of MCR still requires further studies. Moreover, although the functions of PTMs are indicated to be related to the catalysis and stability of MCR, no consistency has been given on the exact functions of each PTM. Furthermore, the results of functional studies of PTMs may vary in different species, which have different growth features.

\section{AUTHOR CONTRIBUTIONS}

HC, QG, and CF wrote and edited the manuscript. All authors contributed to the article and approved the submitted version.

\section{FUNDING}

This work was supported by the University of Arkansas. 
Ermler, U., Grabarse, W., Shima, S., Goubeaud, M., and Thauer, R. K. (1997). Crystal structure of methyl-coenzyme $\mathrm{M}$ reductase: the key enzyme of biological methane formation. Science 278, 1457-1462. doi: 10.1126/ science.278.5342.1457

Evans, P. N., Boyd, J. A., Leu, A. O., Woodcroft, B. J., Parks, D. H., Hugenholtz, P., et al. (2019). An evolving view of methane metabolism in the archaea. Nat. Rev. Microbiol. 17, 219-232. doi: 10.1038/s41579-018-0136-7

Goubeaud, M., Schreiner, G., and Thauer, R. K. (1997). Purified methylcoenzyme-M reductase is activated when the enzyme-bound coenzyme F430 is reduced to the nickel(I) oxidation state by titanium(III) citrate. Eur. J. Biochem. 243, 110-114. doi: 10.1111/j.1432-1033.1997.00110.x

Grabarse, W., Mahlert, F., Duin, E. C., Goubeaud, M., Shima, S., Thauer, R. K., et al. (2001). On the mechanism of biological methane formation: structural evidence for conformational changes in methyl-coenzyme M reductase upon substrate binding. J. Mol. Biol. 309, 315-330. doi: 10.1006/jmbi. 2001.4647

Grabarse, W., Mahlert, F., Shima, S., Thauer, R. K., and Ermler, U. (2000). Comparison of three methyl-coenzyme $\mathrm{M}$ reductases from phylogenetically distant organisms: unusual amino acid modification, conservation and adaptation. J. Mol. Biol. 303, 329-344. doi: 10.1006/jmbi.2000.4136

Harmer, J., Finazzo, C., Piskorski, R., Bauer, C., Jaun, B., Duin, E. C., et al. (2005). Spin density and coenzyme M coordination geometry of the ox1 form of methyl-coenzyme M reductase: a pulse EPR study. J. Am. Chem. Soc. 127, 17744-17755. doi: 10.1021/ja053794w

Horng, Y. -C., Becker, D. F., and Ragsdale, S. W. (2001). Mechanistic studies of methane biogenesis by methyl-coenzyme $M$ reductase: evidence that coenzyme B participates in cleaving the $\mathrm{C}-\mathrm{S}$ bond of methyl-coenzyme $\mathrm{M}$. Biochemistry 40, 12875-12885. doi: 10.1021/bi011196y

Kahnt, J., Buchenau, B., Mahlert, F., Krüger, M., Shima, S., and Thauer, R. K. (2007). Post-translational modifications in the active site region of methylcoenzyme $M$ reductase from methanogenic and methanotrophic archaea. FEBS J. 274, 4913-4921. doi: 10.1111/j.1742-4658.2007.06016.x

Kirschke, S., Bousquet, P., Ciais, P., Saunois, M., Canadell, J. G., Dlugokencky, E. J., et al. (2013). Three decades of global methane sources and sinks. Nat. Geosci. 6, 813-823. doi: 10.1038/ngeo 1955

Knittel, K., and Boetius, A. (2009). Anaerobic oxidation of methane: progress with an unknown process. Annu. Rev. Microbiol. 63, 311-334. doi: 10.1146/ annurev.micro.61.080706.093130

Liscombe, D. K., Louie, G. V., and Noel, J. P. (2012). Architectures, mechanisms and molecular evolution of natural product methyltransferases. Nat. Prod. Rep. 29, 1238-1250. doi: 10.1039/c2np20029e

Lyu, Z., Chou, C. -W., Shi, H., Wang, L., Ghebreab, R., Phillips, D., et al. (2018a). Assembly of methyl coenzyme M reductase in the methanogenic archaeon Methanococcus maripaludis. J. Bacteriol. 200, e00746-e00717. doi: 10.1128/JB.00746-17

Lyu, Z., Shao, N., Akinyemi, T., and Whitman, W. B. (2018b). Methanogenesis. Curr. Biol. 28, R727-R732. doi: 10.1016/j.cub.2018.05.021

Lyu, Z., Shao, N., Chou, C. -W., Shi, H., Patel, R., Duin, E. C., et al. (2020). Posttranslational methylation of arginine in methyl coenzyme $\mathrm{M}$ reductase has a profound impact on both methanogenesis and growth of Methanococcus maripaludis. J. Bacteriol. 202, e00654-e00719. doi: 10.1128/JB.00654-19

Lyu, Z., and Whitman, W. B. (2019). Transplanting the pathway engineering toolbox to methanogens. Curr. Opin. Biotechnol. 59, 46-54. doi: 10.1016/j. copbio.2019.02.009

Mahanta, N., Liu, A., Dong, S., Nair, S. K., and Mitchell, D. A. (2018). Enzymatic reconstitution of ribosomal peptide backbone thioamidation. Proc. Natl. Acad. Sci. U. S. A. 115, 3030-3035. doi: 10.1073/pnas.1722324115

Mansoorabadi, S. O., Zheng, K., and Ngo, P. D. (2017). "Biosynthesis of coenzyme F430 and the posttranslational modification of the active site region of methyl-coenzyme $\mathrm{M}$ reductase" in Metalloprotein active site assembly. eds. M. Johnson and R. Scott (Wiley), 323.

Nayak, D. D., Liu, A., Agrawal, N., Rodriguez-Carerro, R., Dong, S. -H., Mitchell, D. A., et al. (2020). Functional interactions between posttranslationally modified amino acids of methyl-coenzyme M reductase in Methanosarcina acetivorans. PLoS Biol. 18:e3000507. doi: 10.1371/journal.pbio.3000507

Nayak, D. D., Mahanta, N., Mitchell, D. A., and Metcalf, W. W. (2017). Posttranslational thioamidation of methyl-coenzyme $\mathrm{M}$ reductase, a key enzyme in methanogenic and methanotrophic archaea. Elife 6:e29218. doi: 10.7554/ eLife. 29218
Orphan, V. J., House, C. H., Hinrichs, K. -U., McKeegan, K. D., and DeLong, E. F. (2001). Methane-consuming archaea revealed by directly coupled isotopic and phylogenetic analysis. Science 293, 484-487. doi: 10.1126/science. 1061338

Prakash, D., Wu, Y., Suh, S. -J., and Duin, E. C. (2014). Elucidating the process of activation of methyl-coenzyme M reductase. J. Bacteriol. 196, 2491-2498. doi: 10.1128/JB.01658-14

Radle, M. I., Miller, D. V., Laremore, T. N., and Booker, S. J. (2019). Methanogenesis marker protein 10 (Mmp10) from Methanosarcina acetivorans is a radical S-adenosylmethionine methylase that unexpectedly requires cobalamin. J. Biol. Chem. 294, 11712-11725. doi: 10.1074/jbc.RA119.007609

Ragsdale, S. W. (2014). "Biochemistry of methyl-coenzyme M reductase: the nickel metalloenzyme that catalyzes the final step in synthesis and the first step in anaerobic oxidation of the greenhouse gas methane" in The metaldriven biogeochemistry of gaseous compounds in the environment. eds. P. Kroneck and M. S. Torres (Netherlands: Springer), 125-145.

Rospert, S., Böcher, R., Albracht, S. P. J., and Thauer, R. K. (1991). Methylcoenzyme $M$ reductase preparations with high specific activity from $\mathrm{H} 2$ preincubated cells of Methanobacterium thermoautotrophicum. FEBS Lett. 291, 371-375. doi: 10.1016/0014-5793(91)81323-Z

Rospert, S., Linder, D., Ellermann, J., and Thauer, R. K. (1990). Two genetically distinct methyl-coenzyme $\mathrm{M}$ reductases in Methanobacterium thermoautotrophicum strain Marburg and $\Delta$ H. Eur. J. Biochem. 194, 871-877. doi: 10.1111/j.14321033.1990.tb19481.x

Scheller, S., Goenrich, M., Boecher, R., Thauer, R. K., and Jaun, B. (2010). The key nickel enzyme of methanogenesis catalyses the anaerobic oxidation of methane. Nature 465, 606-608. doi: 10.1038/nature09015

Scheller, S., Goenrich, M., Thauer, R. K., and Jaun, B. (2013). Methyl-coenzyme $\mathrm{M}$ reductase from methanogenic archaea: isotope effects on the formation and anaerobic oxidation of methane. J. Am. Chem. Soc. 135, 14975-14984. doi: $10.1021 /$ ja406485z

Scheller, S., Yu, H., Chadwick, G. L., McGlynn, S. E., and Orphan, V. J. (2016). Artificial electron acceptors decouple archaeal methane oxidation from sulfate reduction. Science 351, 703-707. doi: 10.1126/science.aad7154

Selmer, T., Kahnt, J., Goubeaud, M., Shima, S., Grabarse, W., Ermler, U., et al. (2000). The biosynthesis of methylated amino acids in the active site region of methyl-coenzyme M reductase. J. Biol. Chem. 275, 3755-3760. doi: 10.1074/ jbc.275.6.3755

Shima, S., Krueger, M., Weinert, T., Demmer, U., Kahnt, J., Thauer, R. K., et al. (2012). Structure of a methyl-coenzyme M reductase from Black Sea mats that oxidize methane anaerobically. Nature 481, 98-101. doi: 10.1038/ nature 10663

Sieber, J. R., McInerney, M. J., and Gunsalus, R. P. (2012). Genomic insights into syntrophy: the paradigm for anaerobic metabolic cooperation. Annu. Rev. Microbiol. 66, 429-452. doi: 10.1146/annurev-micro-090110102844

Thauer, R. K. (2019). Methyl (alkyl)-coenzyme M reductases: nickel F-430containing enzymes involved in anaerobic methane formation and in anaerobic oxidation of methane or of short chain alkanes. Biochemistry 58, 5198-5220. doi: 10.1021/acs.biochem.9b00164

Timmers, P. H. A., Welte, C. U., Koehorst, J. J., Plugge, C. M., Jetten, M. S. M., and Stams, A. J. M. (2017). Reverse methanogenesis and respiration in methanotrophic archaea. Archaea 2017:1654237. doi: 10.1155/2017/ 1654237

Vanwonterghem, I., Evans, P. N., Parks, D. H., Jensen, P. D., Woodcroft, B. J., Hugenholtz, P., et al. (2016). Methylotrophic methanogenesis discovered in the archaeal phylum Verstraetearchaeota. Nat. Microbiol. 1:16170. doi: 10.1038/ nmicrobiol.2016.170

Wagner, T., Kahnt, J., Ermler, U., and Shima, S. (2016). Didehydroaspartate modification in methyl-coenzyme $\mathrm{M}$ reductase catalyzing methane formation. Angew. Chem. Int. Ed. Engl. 55, 10630-10633. doi: 10.1002/ anie. 201603882

Wagner, T., Wegner, C. -E., Kahnt, J., Ermler, U., and Shima, S. (2017). Phylogenetic and structural comparisons of the three types of methyl coenzyme M reductase from Methanococcales and Methanobacteriales. J. Bacteriol. 199, e00197-e00117. doi: 10.1128/JB.00197-17

Wang, F. -P., Zhang, Y., Chen, Y., He, Y., Qi, J., Hinrichs, K. -U., et al. (2014). Methanotrophic archaea possessing diverging methane-oxidizing and electrontransporting pathways. ISME J. 8, 1069-1078. doi: 10.1038/ismej.2013.212 
Wongnate, T., and Ragsdale, S. W. (2015). The reaction mechanism of methylcoenzyme $M$ reductase: how an enzyme enforces strict binding order. J. Biol. Chem. 290, 9322-9334. doi: 10.1074/jbc.M115.636761

Wongnate, T., Sliwa, D., Ginovska, B., Smith, D., Wolf, M. W., Lehnert, N., et al. (2016). The radical mechanism of biological methane synthesis by methyl-coenzyme M reductase. Science 352, 953-958. doi: 10.1126/science. aaf0616

Zhou, Y., Dorchak, A. E., and Ragsdale, S. W. (2013). In vivo activation of methyl-coenzyme M reductase by carbon monoxide. Front. Microbiol. 4:69. doi: $10.3389 /$ fmicb.2013.00069
Conflict of Interest: The authors declare that the research was conducted in the absence of any commercial or financial relationships that could be construed as a potential conflict of interest.

Copyright (c) 2020 Chen, Gan and Fan. This is an open-access article distributed under the terms of the Creative Commons Attribution License (CC BY). The use, distribution or reproduction in other forums is permitted, provided the original author(s) and the copyright owner(s) are credited and that the original publication in this journal is cited, in accordance with accepted academic practice. No use, distribution or reproduction is permitted which does not comply with these terms. 